\title{
Identification of candidate target genes of pituitary adenomas based on the DNA microarray
}

\author{
WEI ZHOU, CHUN-XIAO MA, YA-ZHOU XING and ZHAO-YUE YAN \\ Department of Neurosurgery, Henan Provincial People's Hospital, Zhengzhou, Henan 450003, P.R. China
}

Received December 16, 2014; Accepted September 3, 2015

DOI: $10.3892 / \mathrm{mmr} .2016 .4785$

\begin{abstract}
The present study aimed to explore molecular mechanisms involved in pituitary adenomas (PAs) and to discover target genes for their treatment. The gene expression profile GSE4488 was downloaded from the Gene Expression Omnibus database. Differentially expressed genes (DEGs) were identified using the Limma package and analyzed by two-dimensional hierarchical clustering. Gene ontology (GO) and pathway enrichment analyses were performed in order to investigate the functions of DEGs. Subsequently, the protein-protein interaction (PPI) network was constructed using Cytoscape software. DEGs were then mapped to the connectivity map database to identify molecular agents associated with the underlying mechanisms of PAs. A total of 340 upregulated and 49 downregulated DEGs in PA samples compared with those in normal controls were identified. Hierarchical clustering analysis showed that DEGs were highly differentially expressed, indicating their aptness for distinguishing PA samples from normal controls. Significant gene ontology terms were positive regulation of immune system-associated processes for downregulated DEGs and skeletal system development for upregulated DEGs. Pathways significantly enriched by DEGs included extracellular matrix (ECM)-receptor interaction, the Hedgehog ( $\mathrm{Hh}$ ) signaling pathway and neuroactive ligand-receptor interaction. The PPI network was constructed with 117 nodes, 123 edges and CD44 and Gli2 as hub nodes. Furthermore, depudecin, a small molecule drug, was identified to be mechanistically associated with PA. The genes CD44 and Gli2 have important roles in the progression of PAs via ECM-receptor interaction and the Hh signaling pathway and are therefore potential target genes of PA. In addition, depudecin may be a candidate drug for the treatment of PAs.
\end{abstract}

Correspondence to: Dr Wei Zhou, Department of Neurosurgery, Henan Provincial People's Hospital, 7 Weiwu Road, Zhengzhou, Henan 450003, P.R. China

E-mail: weizhouzhh@163.com

Key words: pituitary adenomas, differentially expressed genes, pathway analysis, protein-protein interaction network analysis

\section{Introduction}

Pituitary adenomas (PAs) are common benign neoplasms and $\sim 10-25 \%$ being intracranial neoplasms. Cross-sectional studies from Switzerland, Belgium and the UK have shown that PAs have a prevalence of 78-94 cases/100,000 inhabitants (1). PAs are formed due to hypersecretion/hyposecretion of a number of or all of the pituitary hormones and/or due to local tumor compression (2). The vast majority of PAs occur sporadically; however, familial cases are now increasingly recognized (2). Presently used drugs, including metyrapone (3), ketoconazole (4) and mitotane (5), inhibit the secretion and synthesis of cortisol, which is associated with PAs in the adrenal gland. However, due to their side effects and moderate efficacy, these drugs have limitations in PA treatment (5-7). Therefore, it is required to elucidate the underlying molecular mechanisms of PAs in order to discover novel targets and potential drugs for their treatment.

It is commonly thought that the occurrence and development of PAs is due to abberant gene expression in pituitary cells as well as hypothalamic dysfunction (8). It has been reported that Glil, which is activated by the hedgehog (Hh) signal transduction cascade, has a crucial role in the pathogenesis of PAs by modulating adult stem cell fate or tumor-initiating stem cell function in the adult pituitary gland and its neoplasms (9). In addition, Cazabat et al (10) suggested that aryl hydrocarbon receptor-interacting protein, which is a ligand-activated transcription factor found in the cytoplasm, is associated with the occurrence of PAs. Furthermore, a study reported that the neuroactive ligand-receptor interaction signaling pathway is associated with the development of PAs (11). Although great efforts have been made to explore the pathogenesis of PAs and discover novel target genes for PA treatment, the current knowledge is insufficient.

To obtain a systematic perspective for understanding the underlying mechanisms of PAs and to discover novel therapeutic targets for PA treatment, the present study utilized bioinformatics methods to analyze gene expression profiles and performed functional analysis of differentially expressed genes (DEGs) between PA samples and normal controls. Furthermore, the protein-protein interaction (PPI) network was constructed to identify hub genes associated with PAs and small molecular drugs with associated mechanisms were screened. The present study provided a basis for exploring the potential underlying molecular mechanism of PAs and to discover candidate target genes for PA treatment. 


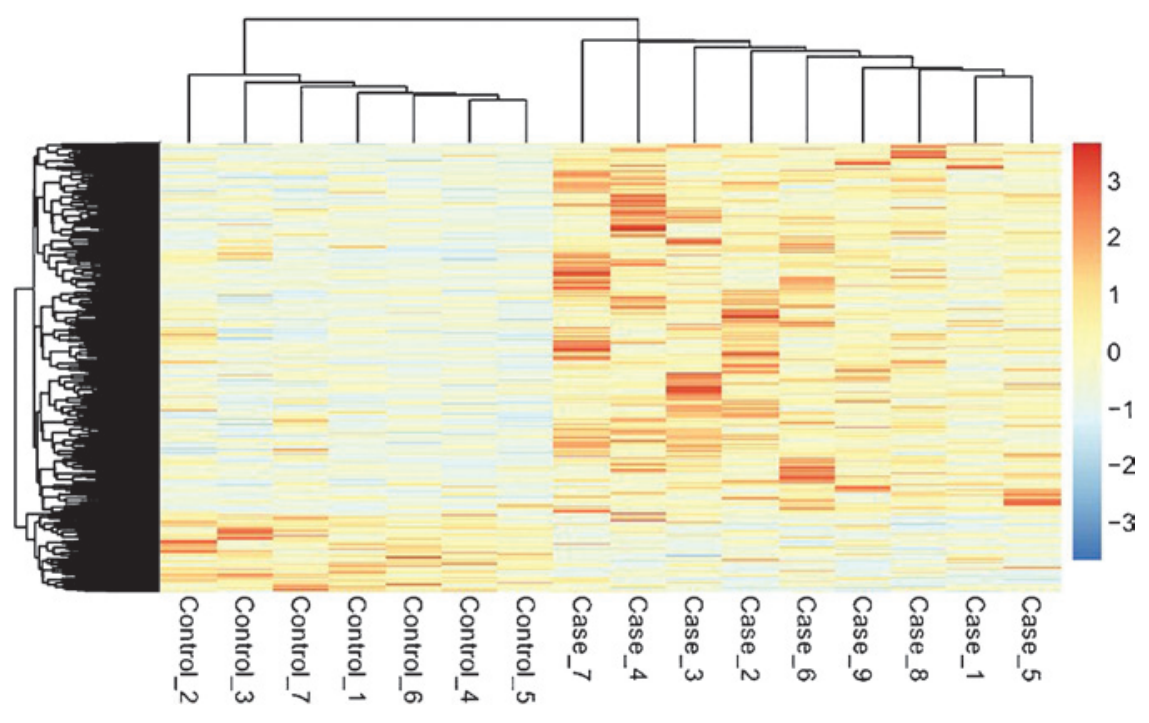

Figure 1. Hierarchical clustering of differentially expressed genes.

\section{Materials and methods}

Affymetrixmicroarray data and data pre-processing. The array dataset GSE4488 was downloaded from the Gene Expression Omnibus (GEO) database from the national center of biotechnology information (http://www.ncbi.nlm.nih.gov/geo/), which was deposited by Vierimaa et al (12). The expression profiles analyzed in the present study had been obtained from nine blood samples from patients with PAs and seven blood samples from healthy controls. The platform via which the data had been obtained was the GPL570 (Affymetrix Human Genome U133 Plus 2.0 Array; Affymetrix, Inc., Santa Clara, CA, USA). All of the array data were pre-processed using the robust multi-array average algorithm (13). Normalization was performed at probe level. Whenever multiple probes corresponded to the same gene, the mean value was calculated as the gene expression value for this gene.

Screening of DEGs and hierarchical clustering analysis. The Limma package (14) in R language was used to screen DEGs. DEGs with $\mid \log 2$ fold change $(\mathrm{FC}) \mid>1$ and $\mathrm{P}<0.05$ were considered to be significant.

Hierarchical cluster analysis produces a unique set of nested categories or clusters by sequentially pairing variables, clusters, or variables and clusters (15). The gene expression profiles of the selected DEGs was subjected to two-dimensional hierarchical clustering analysis based on Euclidean distance using the 'pheatmap' package in R language (16) and then the heat map was generated.

Gene ontology (GO) and pathway enrichment analysis. GO analysis is a commonly used approach for functional studies of genomic or transcriptomic data (17). In order to analyze the DEGs at the function level, GO annotation for DEGs was performed using the online software Database for Annotation, Visualization and Integration Discovery (18). The DEGs were classified into three GO categories, including molecular function, biological process and cellular components. $\mathrm{P}<0.05$ was set as the threshold value.
The Kyoto Encyclopedia of Genes and Genomes (KEGG) knowledge database is used for classification of correlating gene sets into their respective pathways (19). The Web-based Gene Set Analysis Toolkit (http://bioinfo.vanderbilt. edu/webgestalt/WebGestalt) $(20,21)$ was applied for the enrichment tests of KEGG pathways. $\mathrm{P}<0.05$ was selected as the threshold.

PPI network construction. The Search Tool for the Retrieval of Interacting Genes (STRING) (22) is an online database resource, which collects comprehensive information of predicted and experimental interactions of proteins. The interactions of protein pairs in the STRING database were displayed with a combined score $>0.4$. The PPI network with significant gene pairs was then visualized using Cytoscape software (23).

Identification of candidate agents. The Connectivity Map (cMap) $(24,25)$ database collects the gene expression profiles from cultured human cells treated with small molecules. The DEGs were converted into a probe set on the GPL570 platform and mapped onto the cMap database. Small molecules mechanistically associated with PAs were then identified as candidate agents using thresholds of Iconnectivity scorel $>0.8$ and $\mathrm{P}<0.05$.

\section{Results}

Screening of DEGs and hierarchical clustering analysis. According to the cut-off criteria of $\mathrm{P}<0.05$ and $\log 2 \mathrm{FCl}>2.0$, a total of 389 DEGs were obtained, including 49 downregulated and 340 upregulated DEGs.

The heat map of hierarchical clustering analysis for the DEGs is shown in Fig. 1, which clearly demonstrated the obvious differences in expression between PAs and normal controls. The expression values for the same gene in the two groups were significantly different, rendering the DEGs suitable for distinguishing PAs from normal controls.

GO and pathway enrichment analysis. The results of the GO analysis are shown in Table I. The upregulated DEGs 
Table I. Gene ontology enrichment analysis for differentially expressed genes.

\begin{tabular}{|c|c|c|c|}
\hline Gene ontology term & Function & Count & P-value \\
\hline \multicolumn{4}{|l|}{ Downregulated } \\
\hline 0048584 & Positive regulation of response to stimulus & 4 & $1.79 \times 10^{-2}$ \\
\hline 0002684 & Positive regulation of immune system process & 4 & $1.83 \times 10^{-2}$ \\
\hline 0002521 & Leukocyte differentiation & 3 & $3.82 \times 10^{-2}$ \\
\hline 0050926 & Regulation of positive chemotaxis & 2 & $4.40 \times 10^{-2}$ \\
\hline 0050927 & Positive regulation of positive chemotaxis & 2 & $4.40 \times 10^{-2}$ \\
\hline 0010628 & Positive regulation of gene expression & 5 & $4.68 \times 10^{-2}$ \\
\hline \multicolumn{4}{|l|}{ Upregulated } \\
\hline 0007588 & Excretion & 5 & $9.52 \times 10^{-3}$ \\
\hline 0060429 & Epithelium development & 9 & $1.67 \times 10^{-2}$ \\
\hline 0007398 & Ectoderm development & 8 & $2.47 \times 10^{-2}$ \\
\hline 0009314 & Response to radiation & 8 & $2.53 \times 10^{-2}$ \\
\hline 0007200 & Activation of phospholipase $\mathrm{C}$ activity & 4 & $3.84 \times 10^{-2}$ \\
\hline 0045664 & Regulation of neuron differentiation & 6 & $4.26 \times 10^{-2}$ \\
\hline 0015698 & Inorganic anion transport & 5 & $4.47 \times 10^{-2}$ \\
\hline 0009416 & Response to light stimulus & 6 & $4.86 \times 10^{-2}$ \\
\hline
\end{tabular}

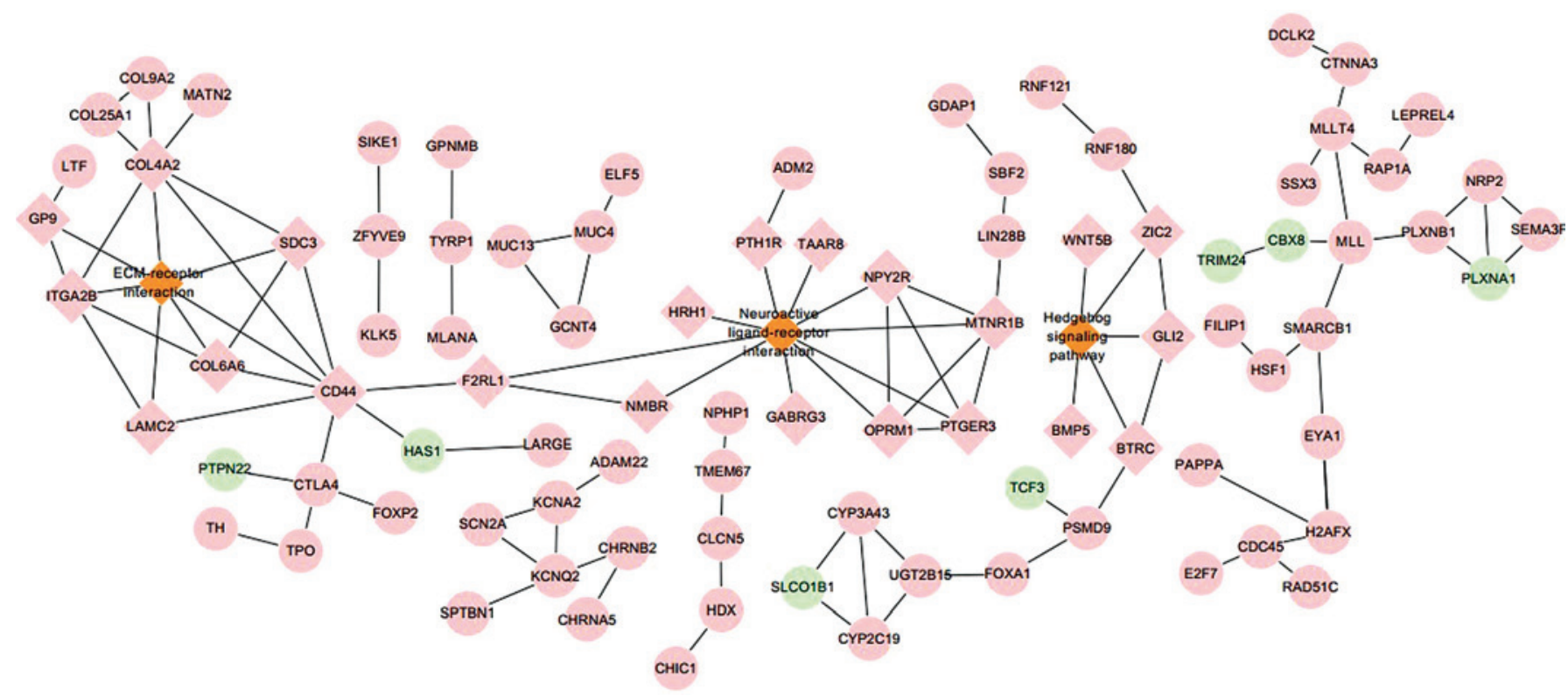

Figure 2. Protein-protein interaction network of DEGs. Red nodes, upregulated genes; green nodes, downregulated genes; orange rhombi, Kyoto Encyclopedia of Genes and Genomes pathways; rhombic nodes with red or green, DEGs involved in pathways. ECM, extracellular matrix; DEG, differentially expressed gene.

were mainly enriched in ectodermal development, epithelial development, response to radiation and skeletal system development, while the downregulated DEGs were mainly involved in responses to stimuli, immune system-associated processes and gene expression.

Furthermore, only three significant pathways of the screened DEGs were enriched, including extracellular matrix (ECM)-receptor interaction [Homo sapiens (hsa)04512], the Hh signaling pathway (hsa04340) and neuro-active ligand-receptor interaction (hsa04080) (Table II).

PPI network construction and functional analysis of DEGs. Based on the STRING database, a total of 101 gene pairs with a combined score $>0.4$ were obtained. The PPI network shown in Fig. 2 contained three pathways comprising 117 nodes and 123 edges. The hub proteins of cluster of differentiation 44 (CD44) and laminin, gamma 2 (LAMC2) were involved in the ECM-receptor interaction pathway, while prostaglandin E receptor 3 (PTGER3) was associated with the neuroactive ligand-receptor interaction pathway. Glioma-associated oncogene 2 (Gli2), which took part in the hedgehog signaling pathway, was also a hub protein in the PPI network.

Screening of small molecular drug candidates. After mapping of DEGs onto the cMap database, a total of 13 potentially mechanistically associated small molecules were obtained, 
Table II. Enriched Kyoto Encyclopedia of Genes and Genomes pathway of differentially expressed genes.

\begin{tabular}{llc}
\hline ID & \multicolumn{1}{c}{ Pathway } & P-value \\
\hline hsa04512 & ECM-receptor interaction & $2.47 \times 10^{-3}$ \\
hsa04340 & Hedgehog signaling pathway & $1.33 \times 10^{-2}$ \\
hsa04080 & Neuroactive ligand-receptor & $2.44 \times 10^{-2}$ \\
& interaction & \\
\hline
\end{tabular}

hsa, Homo sapiens; ECM, extracellular matrix.

Table III. Enriched significant small molecules.

\begin{tabular}{lcc}
\hline cMap name & Enrichment & P-value \\
\hline Depudecin & -0.935 & $8.71 \times 10^{-3}$ \\
Sulfamonomethoxine & -0.863 & $6.40 \times 10^{-4}$ \\
Sulfadimethoxine & -0.807 & $6.20 \times 10^{-4}$ \\
Prestwick-692 & -0.806 & $2.71 \times 10^{-3}$ \\
Podophyllotoxin & -0.803 & $2.88 \times 10^{-3}$ \\
Cefamandole & -0.801 & $3.06 \times 10^{-3}$ \\
Verteporfin & 0.802 & $1.58 \times 10^{-2}$ \\
Thioguanosine & 0.803 & $2.84 \times 10^{-3}$ \\
Fluorocurarine & 0.804 & $2.80 \times 10^{-3}$ \\
Anabasine & 0.811 & $1.36 \times 10^{-2}$ \\
Blebbistatin & 0.858 & $4.11 \times 10^{-2}$ \\
Imidurea & 0.918 & $1.14 \times 10^{-3}$ \\
Spiperone & 0.973 & $1.11 \times 10^{-3}$ \\
\hline
\end{tabular}

which are listed in Table III. The small molecule depudecin (connectivity score, -0.935) had the highest negative score.

\section{Discussion}

PAs are a common type of benign intracranial neoplasm (26). In previous studies, gene expression profiling has been used to identify germline mutations associated with the pre-disposition to pituitary adenoma (26). KEGG pathway enrichment analysis performed in the present study showed that DEGs were significantly enriched in ECM-receptor interaction and Hh signaling pathways, including $C D 44$ and $G l i 2$, which were hub proteins in the PPI network. Furthermore, the small-molecule depudecin was identified as a potential drug for the to treatment of PAs.

The present study identified that CD44 was a hub protein in the PPI network and had a significant role in the ECM-receptor interaction pathway (27). CD44 is a cell-surface glycoprotein (28). It is a receptor for hyaluronic acid (HA) and can interact with ligands such as osteopontin, collagens and matrix metalloproteinases (29-31). It is involved in cellular functions, including cell-cell interactions, cell adhesion and cell migration (31). Upregulation of CD44 may induce signaling events that promote anchorage-independent tumor-cell growth, survival and migration, thereby increasing metastatic spread $(32,33)$. Previous studies have reported that CD44 is expressed in PAs (34) and that its expression levels are significantly upregulated in PAs (35). However, CD44 is also an ECM receptor, which has a crucial role in tumorigenesis. It has been reported that pituitary tumorigenesis involves remodeling of the ECM (36). The CD44-ECM interaction can contribute to malignant transformation in an indirect manner, for instance by regulating sensitivity to inflammation (37). The cleavage of CD44 and ECM can promote cell migration of pituitary adenoma (38); therefore, CD44 may induce signaling events and interact with the ECM to promote the tumorigenesis of pituitary adenoma.

Gli2, which has a key role in pituitary development, belongs to the $\mathrm{C} 2 \mathrm{H} 2$-type zinc finger protein sub-class of the Gli family. The members of the Gli family are mediators of the Sonic hedgehog (Shh) signaling pathway. A previous study has shown that loss-of-function mutations in the human Gli2 gene are associated with PAs (39). Furthermore, Devine et al (40) indicated that dysregulation of Gli2 function may contribute to PAs. Hh signaling is necessary for the induction and functional patterning of the pituitary placode (40). The pathway analysis of the present study showed that Gli2 is an important member of this pathway. A previous study showed that downregulation of Shh, which is a member of the Hh family, increases the proliferation of PA cells and may be involved in the pathogenesis of PAs (41). Gli2 is the most important mediator of Shh signaling in vertebrates (42). It is required for repressing Shh gene expression posteriorly in the pars intermedia (40). Accordingly, Gli2 takes part in the development of PAs through the Hh signaling pathway.

In addition, the present study discovered candidate small molecules which may be implicated the development of PAs (positive cMAP enrichment factor) or which may be suitable drugs for the treatment of PAs (negative cMAP enrichment factor). Depudecin was identified to be small molecule drug with the most significant reverse mechanistic association with PAs. Depudecin is a fungal metabolite containing two epoxide groups (43). It was reported to have anti-angiogenic activity (44), regulate the assembly of the actin microfilament components of the cytoskeleton in mammalian cells (45) and to induces morphological reversion of transformed fibroblasts (46). Depudecin has been patented as a histone deacetylase inhibitor for the treatment of neuroendocrine tumors (47). The present study indicated a close reverse mechanistic association of depudecin with PAs, therefore suggesting that it may be a suitable drug for its treatment; however, the efficacy of depudecin against PAs as well as its mechanism of action remain to be elucidated in future studies.

In conclusion, the present study identified enriched pathways in PAs, generated a PPI network and virtually screened candidate small molecules associated with PAs. The genes CD44 and Gli2, which are involved in ECM-receptor interactions and $\mathrm{Hh}$ signaling, were shown to have significant roles in the development of PAs. In addition, depudecin may be a candidate drug for treating PAs. The present study provided a systematic perspective to elucidate the underlying mechanism of PAs, including molecular targets for their treatment. However, the present study was performed using bioinformatics methods and the conclusions remain to be confirmed by corresponding experiments. Therefore, further study is required to verify the underlying mechanisms of metastatic PAs. 


\section{References}

1. Karavitaki N: Prevalence and incidence of pituitary adenomas. Ann Endocrinol (Paris) 73: 79-80, 2012.

2. Aflorei ED and Korbonits M: Epidemiology and etiopathogenesis of pituitary adenomas. J Neurooncol 117: 379-394, 2014.

3. Kokshoorn NE, Romijn JA, Roelfsema F, Rambach AH, Smit JW, Biermasz NR and Pereira AM: The use of an early postoperative $\mathrm{CRH}$ test to assess adrenal function after transsphenoidal surgery for pituitary adenomas. Pituitary 15: 436-444, 2012

4. Feelders RA, de Bruin C, Pereira AM, Romijn JA, Netea-Maier RT, Hermus AR, Zelissen PM, van Heerebeek R, de Jong FH, van der Lely AJ, et al: Pasireotide alone or with cabergoline and ketoconazole in Cushing's disease. N Engl J Med 362: 1846-1848, 2010.

5. Biller BM, Grossman AB, Stewart PM, Melmed S, Bertagna X, Bertherat J, Buchfelder M, Colao A, Hermus AR, Hofland LJ, et al: Treatment of adrenocorticotropin-dependent Cushing's syndrome: A consensus statement. J Clin Endocrinol Metab 93: 2454-2462, 2008.

6. Newell-Price J, Bertagna X, Grossman AB and Nieman LK: Cushing's syndrome. Lancet 367: 1605-1617, 2006.

7. Tritos NA, Biller BM and Swearingen B: Management of Cushing disease. Nat Rev Endocrinol 7: 279-289, 2011.

8. Gong J, Diao B, Yao GJ, Liu Y and Xu GZ: Analysis of regulatory networks constructed based on gene coexpression in pituitary adenoma. J Genet 92: 489-497, 2013.

9. Lampichler K, Ferrer P, Vila G, et al: The role of GLI1 in pituitary tumor formation and pituitary cell survival. 2013.

10. Cazabat L, Bouligand $\mathrm{J}$ and Chanson P: AIP mutation in pituitary adenomas. N Engl J Med 364: 1973-1974, 2011.

11. Riester A, Issler O, Spyroglou A, Rodrig SH, Chen A and Beuschlein F: ACTH-dependent regulation of microRNA as endogenous modulators of glucocorticoid receptor expression in the adrenal gland. Endocrinology 153: 212-222, 2012.

12. Vierimaa O, Georgitsi M, Lehtonen R, Vahteristo P, Kokko A, Raitila A, Tuppurainen K, Ebeling TM, Salmela PI, Paschke R, et al: Pituitary adenoma predisposition caused by germline mutations in the AIP gene. Science 312: 1228-1230, 2006.

13. Irizarry RA, Hobbs B, Collin F, Beazer-Barclay YD, Antonellis KJ, Scherf U and Speed TP: Exploration, normalization and summaries of high density oligonucleotide array probe level data. Biostatistics 4: 249-264, 2003.

14. Smyth GK: Linear models and empirical bayes methods for assessing differential expression in microarray experiments. Stat Appl Genet Mol Biol 3: Article3, 2004.

15. BRIDGES JRCC: Hierarchical cluster analysis. Psychological reports 18: 851-854, 1966.

16. Team RC: R: A language and environment for statistical computing. 2012.

17. Hulsegge I, Kommadath A and Smits MA: Globaltest and GOEAST: Two different approaches for gene ontology analysis. BMC Proc (3 Suppl 4): S10, 2009.

18. Huang da W, Sherman BT and Lempicki RA: Systematic and integrative analysis of large gene lists using DAVID bioinformatics resources. Nat Protoc 4: 44-57, 2009.

19. Altermann E and Klaenhammer TR: PathwayVoyager: Pathway mapping using the kyoto encyclopedia of genes and genomes (KEGG) database. BMC Genomics 6: 60, 2005.

20. Zhang B, Kirov S and Snoddy J: WebGestalt: An integrated system for exploring gene sets in various biological contexts. Nucleic Acids Res 33: W741-W748, 2005

21. Duncan D, Prodduturi N and Zhang B: WebGestalt2: An updated and expanded version of the Web-based Gene Set Analysis Toolkit. Bmc Bioinformatics 11 (Suppl 4): P10, 2010.

22. Szklarczyk D, Franceschini A, Kuhn M, Simonovic M, Roth A, Minguez P, Doerks T, Stark M, Muller J, Bork P, et al: The STRING database in 2011: Functional interaction networks of proteins, globally integrated and scored. Nucleic Acids Res 39: D561-D568, 2011

23. Kohl M, Wiese S and Warscheid B: Cytoscape: Software for visualization and analysis of biological networks. Methods Mol Biol 696: 291-303, 2011

24. Lamb J: The Connectivity Map: A new tool for biomedical research. Nat Rev Cancer 7: 54-60, 2007.

25. Lamb J, Crawford ED, Peck D, Modell JW, Blat IC, Wrobel MJ, Lerner J, Brunet JP, Subramanian A, Ross KN, et al: The Connectivity Map: Using gene-expression signatures to connect small molecules, genes and disease. Science 313: 1929-1935, 2006.
26. Vierimaa O, Georgitsi M, Lehtonen R, Vahteristo P, Kokko A, Raitila A, Tuppurainen K, Ebeling TM, Salmela PI, Paschke R, et al: Pituitary adenoma predisposition caused by germline mutations in the AIP gene. Science 312: 1228-1230, 2006.

27. Nagano O and Saya H: Mechanism and biological significance of CD44 cleavage. Cancer Sci 95: 930-935, 2004

28. Tsukita S, Oishi K, Sato N, Sagara J, Kawai A and Tsukita S: ERM family members as molecular linkers between the cell surface glycoprotein CD44 and actin-based cytoskeletons. J Cell Biol 126: 391-401, 1994.

29. Lesley J. and Hyman R: CD44 can be activated to function as an hyaluronic acid receptor in normal murine T cells. Eur J Immunol 22: 2719-2723, 1992.

30. Weber GF, Ashkar S, Glimcher MJ and Cantor H: Receptor-ligand interaction between CD44 and osteopontin (Eta-1). Science 271: 509-512, 1996.

31. Ponta H, Sherman L and Herrlich PA: CD44: from adhesion molecules to signalling regulators. Nat Rev Mol Cell Biol 4: 33-45, 2003.

32. Toole BP: Hyaluronan: From extracellular glue to pericellular cue. Nat Rev Cancer 4: 528-539, 2004.

33. Jaracz S, Chen J, Kuznetsova LV and Ojima I: Recent advances in tumor-targeting anticancer drug conjugates. Bioorg Med Chem 13: 5043-5054, 2005

34. Frank S, Rihs H-P, Stöcker W, Müller J, Dumont B, Baur X, Schackert HK and Schackert G: Combined detection of CD44 isoforms by exon-specific RT-PCR and immunohistochemistry in primary human brain tumors and brain metastases. Biochem Biophys Res Commun 222: 794-801, 1996.

35. Duan Bo ZH and Li Xinjian: Expression and relationship between CD44 and Ki-67 in invasive pituitary adenomas. Cancer Res Prev Treat 33: 490-492, 2006.

36. Rubinfeld H, Cohen-Kaplan V, Nass D, Ilan N, Meisel S, Cohen ZR, Hadani M, Vlodavsky I and Shimon I: Heparanase is highly expressed and regulates proliferation in $\mathrm{GH}$-secreting pituitary tumor cells. Endocrinology 152: 4562-4570, 2011.

37. Mantovani A, Allavena P, Sica A and Balkwill F: Cancer-related inflammation. Nature 454: 436-444, 2008.

38. Pan Y, Han C, Wang C, Hu G, Luo C, Gan X, Zhang F, Lu Y and Ding X: ADAM10 promotes pituitary adenoma cell migration by regulating cleavage of CD44 and L1. J Mol Endocrinol 49: 21-33, 2012

39. Roessler E, Du YZ, Mullor JL, Casas E, Allen WP, Gillessen-Kaesbach G, Roeder ER, Ming JE, Ruiz i Altaba A and Muenke M: Loss-of-function mutations in the human GLI2 gene are associated with pituitary anomalies and holoprosencephaly-like features. Proc Natl Acad Sci USA 100: 13424-13429, 2003.

40. Devine CA, Sbrogna JL, Guner B, Osgood M, Shen MC and Karlstrom RO: A dynamic Gli code interprets $\mathrm{Hh}$ signals to regulate induction, patterning and endocrine cell specification in the zebrafish pituitary. Dev Biol 326: 143-154, 2009.

41. Vila G, Theodoropoulou M, Stalla J, Tonn JC, Losa M, Renner U, Stalla GK and Paez-Pereda M: Expression and function of sonic hedgehog pathway components in pituitary adenomas: Evidence for a direct role in hormone secretion and cell proliferation. J Clin Endocrinol Metab 90: 6687-6694, 2005.

42. Ruiz i Altaba A, Palma V and Dahmane N: Hedgehog-Gli signalling and the growth of the brain. Nat Rev Neurosci 3: 24-33, 2002.

43. Matsumoto M, Matsutani S, Sugita K, Yoshida H, Hayashi F, Terui Y, Nakai H, Uotani N, Kawamura Y and Matsumoto K: Depudecin: A novel compound inducing the flat phenotype of NIH3T3 cells doubly transformed by ras-and src-oncogene, produced by Alternaria brassicicola. J Antibiot (Tokyo) 45: 879-885, 1992.

44. Oikawa T, Onozawa C, Inose M and Sasaki M: Depudecin, a microbial metabolite containing two epoxide groups, exhibits anti-angiogenic activity in vivo. Biol Pharm Bull 18: 1305-1307, 1995.

45. Shimada J, Kwon HJ, Sawamura M and Schreiber SL: Synthesis and cellular characterization of the detransformation agent, (-)-depudecin. Chem Biol 2: 517-525, 1995.

46. Kwon HJ, Owa T, Hassig CA, Shimada J and Schreiber SL: Depudecin induces morphological reversion of transformed fibroblasts via the inhibition of histone deacetylase. Proc Natl Acad Sci USA 95: 3356-3361, 1998.

47. Chen $\mathrm{H}$ and Kunnimalaiyaan M: Modulating notch1 signaling pathway for treating neuroendocrine tumors. US Patent 8338482 B2. Filed July 20, 2007; issued December 25, 2012. 